\title{
EDITORIAL THE OPENING TOWARD SCIENTIFIC PRODUCTION IN ENGLISH LANGUAGE
}

It may be paradoxical to launch a new publication in Latin America with a name in English, especially when its contents are mostly in Spanish. Moreover, in this language, literature is large, for example Latindex directory contains about 17,073 journals, 2,689 devoted to Arts and Humanities, 9,847 in Social Sciences and 582 in Psychology; although not all of them are in Spanish, most are. For this, it's necessary to explain why this name, especially if we bear in mind that the discussion of language in science is not trivial, because it affects the fluidity of communication, precision and integration of research and society (Gil-Arnao, de Gil, Rivera, \& Molina, 1998).

At the time of designing the editorial project, there was a certain euphoria on the journal to reach many different countries, being English-speaking countries of major interest because of the richness of the intellectual production currently present in them, without, of course, demerits the contribution made in Spanish language, which was highlighted by, for example, Ardila (2004), who is an exceptional player in this contribution, or by Ardila, Pérez-Acosta and Gutiérrez (2005) in relation to psychology of learning, and can be seen in the Latindex directory of journals (Acain \& Lascurain, 2002), as indicated above. In other places, sometimes, in the Academy, they are not used to read literature in Spanish, while it is quite usual that a Hispano-American scientist becomes familiar with the literature in English. Because of this, and the difficulties that might arise when using a dual designation Spanish/English, we chose to use the name in English. Moreover, the inclusion in the journal of contents mostly in Spanish generates contradiction with the journal name and may also suggest that it was chosen arbitrarily. In fact, we are aware of the effort involved for a Latin American researcher to write in English or to translate a work; because of this, the number of them who chooses writing in that language is very small, so that, sometimes, works of a great significance do not reach readers who might be interested. It is for this reason that International Journal of Psychological Research (IJPR) is presented as a publication in both languages; in this connection, IJPR also aims at integrating the scientific output of English-speaking countries, and it is our desire to bridge the gap that sometimes separates the two scientific worlds and that is reflected in some publications. In this regard, the journal aims to be international, not only by the use of the English language (see Buela-Casal, 2001).

Finally, while these arguments justify the bilingual design of the journal, I want to emphasize that IJPR is intended to be a publication with a high quality both editorial and scientific, the opening towards the scientific production in Englishspeaking countries will also help us on this.

\section{REFERENCES}

Alcain, M. D., \& Lascurain, M. L. (2002). Análisis de las revistas latinoamericanas de psicología incluidas en el "Directorio Latindex". Papeles del Psicólogo, 83, 34-43.

Ardila, R. (2004). Psicología latinoamericana: el primer medio siglo. Revista de Psicología Interamericana / Interamerican Journal of Psychology, 38(2), 317-322.

Ardila, R., Pérez-Acosta, A., \& Gutiérrez, G. (2005).Psicología del aprendizaje: Investigación básica publicada en revistas iberoamericanas indexadas. Revista Latinoamericana de Psicologia, 37(3), 595-615.

Buela-Casal, G. (2001). La psicología española y su proyección internacional, el problema del criterio: internacional, calidad y castellano y/o inglés. Papeles delpsicólogo, 79, 53-57.

Gil-Arnao, F., de Gil, E., Rivera, V., \& Molina, J. (1998). El español en los índices SCI y SSCI. Lapso 1981-1995. Interciencia, $23(1), 33-40$.

Fernando Juárez

Universidad de San Buenaventura, Medellín

fernando_juarez2@yahoo.com 


\section{EDITORIAL LA APERTURA HACIA LA PRODUCCIÓN CIENTÍFICA EN HABLA INGLESA}

Puede resultar paradójico iniciar una nueva publicación en Latinoamérica con una denominación en inglés, especialmente cuando sus contenidos son mayoritariamente en lengua española. Además, en esta lengua, la literatura es numerosa, por ejemplo el directorio Latindex contiene alrededor de 17.073 revistas, 2.689 dedicadas a Artes y Humanidades, 9.847 en Ciencias Sociales y 582 en Psicología; aunque no todas están en idioma español, la mayoría sílo están. Por esto, se hace necesario explicar el porqué de esta denominación, especialmente si tenemos en cuenta que el debate del idioma en la ciencia no es trivial, ya que afecta a la fluidez de la comunicación, a la precisión y a la integración de investigación y sociedad (Gil-Arnao, de Gil, Rivera, \& Molina, 1998).

En el momento de diseñar el proyecto editorial, existió una cierta euforia en relación conque la revista alcanzara muy diferentes países, siendo de gran interés los de habla inglesa debido a la riqueza de la producción intelectual existente actualmente en los mismos, sin, por supuesto, demeritar la contribución realizada en idioma español, la cual ha sido puesta de manifiesto, por ejemplo, por Ardila (2004) quien es un protagonista excepcional en esa contribución, o Ardila, Pérez-Acosta y Gutierrez (2005) en relación a la psicología del aprendizaje, y se puede observar en el directorio de revistas Latindex (Acain \& Lascurain, 2002), como se ha indicado. En otros lugares, a veces no se acostumbra, en los círculos académicos, a leer literatura científica en lengua española; mientras que es bastante usual que un científico en hispanoamérica deba familiarizarse con la literatura en inglés. Debido a esto, y a las dificultades que pudieran surgir al utilizar una doble denominación español/ingles, se optó por utilizar la misma en inglés. Por otra parte, la inclusión en la revista de contenidos mayoritariamente en lengua española, genera contradicción con el nombre de la misma y puede también hacer pensar que la denominación se eligió arbitrariamente. En realidad, somos conscientes del esfuerzo que supone para un investigador hispanoamericano la escritura en idioma inglés o la traducción de un trabajo ya escrito; el número de ellos que se decide por escribir en ese idioma es muy pequeño, por lo que, a veces, trabajos, de una gran relevancia, no llegan a aquellos lectores que podrían estar interesados. Es debido a eso, que International Journal of Psychological Research (IJPR) se presenta como una publicación en ambos idiomas. En relación con lo anterior, IJPR tiene como objetivo la incorporación de producción científica procedente de países de habla inglesa, ya que deseamos que no exista esa brecha que, en ocasiones, separa esos dos mundos científicos y que se refleja en las publicaciones. En este sentido, la revista pretende ser internacional, y no solamente por el uso del idioma inglés (ver Buela-Casal, 2001).

Finalmente, mientras que con estos argumentos justifico el diseño bilingüe de la revista, quiero destacar también que IJPR quiere ser una publicación con altos niveles de calidad editorial y científica, para lo cual ayudará también la apertura hacia la producción científica en habla inglesa.

\section{REFERENCIAS}

Alcain, M. D., \& Lascurain, M. L. (2002). Análisis de las revistas latinoamericanas de psicología incluidas en el "Directorio Latindex". Papeles del Psicólogo, 83, 34-43.

Ardila, R. (2004). Psicología latinoamericana: el primer medio siglo. Revista de Psicología Interamericana / Interamerican Journal of Psychology, 38(2), 317-322.

Ardila, R., Pérez-Acosta, A., \& Gutiérrez, G. (2005).Psicología del aprendizaje: Investigación básica publicada en revistas iberoamericanas indexadas. Revista Latinoamericana de Psicología, 37(3), 595-615.

Buela-Casal, G. (2001). La psicología española y su proyección internacional, el problema del criterio: internacional, calidad y castellano y/o inglés. Papeles delpsicólogo, 79, 53-57.

Gil-Arnao, F., de Gil, E., Rivera, V., \& Molina, J. (1998). El español en los índices SCI y SSCI. Lapso 1981-1995. Interciencia, 23(1), 33-40.

Fernando Juárez

Universidad de San Buenaventura, Medellín

fernando_juarez2@yahoo.com 